\title{
Systematic Review of the Effectiveness of Intervention Strategies for Teaching Mathematics to Secondary School Students
}

\author{
Ermioni Dimou \\ Department of Mathematics, Aristotle University of Thessaloniki, Thessaloniki, Greece \\ Email: std134829@ac.eap.gr
}

How to cite this paper: Dimou, E. (2021) Systematic Review of the Effectiveness of Intervention Strategies for Teaching Mathematics to Secondary School Students. Open Access Library Journal, 8: e7392.

https://doi.org/10.4236/oalib.1107392

Received: April 7, 2021

Accepted: May 24, 2021

Published: May 27, 2021

Copyright $\odot 2021$ by author(s) and Open Access Library Inc.

This work is licensed under the Creative Commons Attribution International License (CC BY 4.0).

http://creativecommons.org/licenses/by/4.0/

\begin{abstract}
The present study was conducted as a Master's Thesis in the postgraduate program "Postgraduate Studies in Mathematics" of the Science School of the Greek Open University. A systematic literature review was performed on the strategic intervention in Secondary Education students with learning disabilities. This review has been implemented according to the definition provided in Fink and its structure follows the review's protocol PRISMA Checklist. The systematic literature review resulted in 15 articles that met the inclusion criteria. The aim of this study is to identify the optimal strategic intervention for Secondary Education students with learning disabilities. The synthesis of results has revealed a series of educational strategies that appear to be beneficial to these students, including, among others, explicit instruction and its models, such as graphic organizer and schema-based instruction, CRA and concrete manipulatives. In the discussion section we will analyze the nature and focus of the interventions applied in the reviewed studies, as well as, the fields and topics of mathematics that consisted of the main study subjects in them. Finally, we will discuss the effects, not only on the research, but also on the application of these interventions on Secondary Education students.
\end{abstract}

\section{Subject Areas}

Teaching and Learning Technologies

\section{Keywords}

Instruction Intervention, Secondary Education Students, Mathematics, Learning Disabilities, Systemic Literature Review, Educational

Teaching Methods 


\section{Introduction}

One element of drawing conclusions about the culture of a society is the education it provides to children. In this education, mathematics is a necessary tool for shielding children's thinking. Mathematics, in addition to the role they play as an academic guarantee for the acquisition of scientific knowledge, since it is an object found in multiple scientific fields, contributes to the development of children's typical mathematical abilities. Previous meta-analytic studies showed that the interventions including Corrective Feedback are moderately effective [1].

But the process of learning mathematics is not always easy. Especially if one considers students with learning difficulties, who are called in addition to the difficulty of learning mathematics themselves to overcome their own difficulties and challenges. Many times for these students the traditional teaching method is not the best solution. Thus, the teacher is called to be more flexible and to implement strategic interventions in order to facilitate students with learning difficulties in learning the subject. These educational interventions can be carried out in various and different levels of intervention.

Strategic interventions for the learning of mathematics in secondary education in our country are the subject of this dissertation. Questions such as which strategic intervention is optimal universally and per mathematical field seek answers from the study on this subject. The study is implemented thanks to the systematic bibliographic review that was carried out in search of primary research during the implementation of which a teaching intervention in mathematics was implemented. In conclusion, the present research is a review of the effectiveness of strategic interventions in the teaching of mathematics.

\section{Theoretical Framework}

\subsection{The Philosophy and Nature of Mathematics}

The various perceptions that prevail in society as well as in the scientific community about the nature and role of mathematics, contribute to the formation and development of mathematics curricula in secondary and not only education. The main division about the nature of mathematics is that in one prevailing view mathematics is a dynamically evolving field, while in the other prevailing view mathematics is a static branch, with already known concepts and fundamental principles [2]. The foundations of these two views are many centuries back, in the heyday of mathematics, mainly of mathematical philosophy and geometry, in ancient Greece and specifically in the time of Plato and Aristotle.

Plato even believed that mathematics is not part of our world, "the world of becoming" as he called it, but part of the "world of Being". The "Being" is a world consisting of Forms, the perfect ideas, such as Justice, Beauty, etc. So mathematics in this respect is established, nothing new is invented, only part of this truth is discovered, as a priori knowledge. On the other hand, Aristotle attached more importance to experience for the acquisition of knowledge as well as to "intuition", where mathematicians use abstract skills to reach perfect geometric 
shapes, something they are able to do through their experience and education.

Based on these two views in the 17th century two philosophical currents prevailed. On the one hand were the rationalists, advocates of Plato's views with their main representatives Descartes, Spinoza and Leibniz. They did not believe in the experience gained from the senses, but they attached great importance to the reason that leads to the acquisition of knowledge. On the other hand, there are those who followed the current of empiricism with Locke, Berkeley, Hume and Reid being representatives of this current. The empiricists believed that we could only rely on our senses to try to understand the world.

And we come to a more modern age up to the present, with the two original philosophical views of Plato and Aristotle being discreetly found in today's philosophical currents about the nature of mathematics as the beginning of thought for each current. At the beginning of the last century, three philosophical currents about mathematics prevailed. The first current was calculus with Frege and Russel as their main representatives, which is an attempt to view mathematics through logic. Formalism follows, represented by Hilbert. A current that accepts calculus but at the same time manipulates symbols based on certain rules and formulas, the knowledge of which must precede in order to achieve mathematical knowledge. Then we meet the philosophical current of Intuitionism founded by the Dutch mathematician Brouwer. This current says that mathematics should be constructed only with finite constructive methods based on the theory of natural numbers, which is presented intuitively [2]. Coming to the present day we find the theory of structuralism and modern mathematics.

Structuralism, a name based on the English word structure, has as its main representatives Benacceraff, Hellman, Resnik and Shapiro. Proponents of structuralism describe themselves as realists, but within the current itself there is additional separation. Benacceraff and Hellman do not support the existence of mathematical objects while Resnick and Shapiro identify their existence based on their usefulness in mathematics by defining it as ontological realists. To better understand structuralism let us define as a system a collection of objects, which have some relations with each other. And as a form or structure let us define the abstract form of a system, which emphasizes the reciprocal relations between objects, while ignoring any other feature that does not affect the way in which it relates to other objects in the system. For example, a language is a system of characters, words and sentences with syntactic and semantic relationships between them. So this system can be understood by reading for example a text and observing the syntax in which it is written or the meaning of the words, ignoring among other things the graphic character or whether it has been handwritten in pencil or pen, because they have no relation to the language system. Therefore, we only observe the structures of the system and ignore everything else in it. By this term structuralists argue that mathematics is the productive study of structures themselves.

Since the mid-1950s there has been an attempt to change the curriculum in 
primary and secondary mathematics worldwide, with the main aim of reducing the gap that had been created between mathematics taught in universities with school mathematics, as the great development of mathematics in the 20th century had led to new knowledge in mathematics. This endeavor was called "new mathematics" or "modern mathematics". And it was not just about modernizing the mathematics taught, but also about retraining and preparing for it the mathematics teachers. In the late 1960s and until the 1980s we saw the gradual implementation of these new programs in Europe, the Soviet Union and America. But since the late 1980s we have seen a shift and rejection of these new curricula. "New mathematics" seems to have given more importance to the understanding of mathematical structures, neglecting arithmetic skills and the formation of routine skills.

In the 21 st century, mathematics is an integral part of modern life. They are found in all school curricula of all levels of education, and in a variety of undergraduate, postgraduate and doctoral programs. While knowledge of mathematics is a key qualification in many jobs. In addition, mathematics finds a role in everyday life, as to solve various situations we turn to mathematics, whether realizing it or not. In general, mathematics contributes to the general and wider social education of an individual. But in addition to all of the above, mathematics cultivates the way of thinking. As they enhance the ability for structured, critical and logical thinking, the ability to recognize logical correlations between independent contingencies, as well as the ability for abstract thinking and generalization. Finally, they help to build discipline, clarity, patience and perseverance as well as learning to create a strategy to deal with a problem.

\subsection{The Teaching of Mathematics in Secondary Education}

Traditionally, mathematics is a school subject, which remained a constant pillar in the respective curricula in the midst of the changes that took place and continue to take place in the educational system of our country. With changes in the curriculum, the weekly teaching hours or even the examination process itself, mathematics still dominates as necessary academic knowledge.

In the educational system of our country, mathematics is taught at all levels of education. From pre-school to the last grade of their school education, students are taught mathematics as a continuation of their knowledge in combination with the addition of young people. Of course mathematics is in the curricula of many scientific fields of higher education [3].

Next we will deal with the curriculum of secondary education based on the textbooks of Mathematics and as presented by the Ministry of Education and Religions for the academic year 2019-2020 and published in Greek Government Leaflet (with the documents under no. 144039/ $\Delta 2 / 17-09-2019,143389 / \Delta 2 / 16-09-2019$ and 143431/D2/16-09-2019).

\subsubsection{Algebra}

The field of algebra is one of the leading and fundamental fields of mathematics. 
The basic parts of algebra, such as the study and application of rules and mathematical symbols, the solution of equations, polynomials, vectors, tables, etc. constitute elementary algebra. While the most abstract parts such as groups and rings constitute abstract or modern algebra. Because of its scope, algebra is applied in many fields other than mathematics itself, such as engineering, medicine, physics, and economics. Although rules similar to arithmetic apply, in elementary algebra we talk about their symbolic solution, since we have numbers replaced by a letter-variable. This has led to evidence of a number of types of properties and applications. An example is the method of solving a quadratic equation through a specific typology, by finding a discriminant and through a specific type of finding its roots. Then the use of these non-arithmetic objects with changes or subtraction in their structural properties led to abstract algebra, i.e. in groups, rings and fields.

In high school in our country, students are taught again basic arithmetic rules, mainly the study of fractions, as well as the study of explicit and generally negative numbers. They start from the very first grade of high school to replace the arithmetic factor with a letter. In the next class they are able to solve a first degree equation with a stranger, while having the first contact with basic functions. While in the last grade of high school students enter many new concepts. They study mononyms and polynomials, learn basic mathematical identities, are able to factorize, solve secondary equations with the method of factorization, but also applying methodology using the type of discriminant and finding its roots. They also know how to solve an inequality with a stranger, as well as solve a linear system with two unknowns algebraically in two ways. Finally, they enter the basic knowledge of trigonometry.

Having received basic knowledge on elementary algebra since high school, in senior high school they do a little more in-depth study in this area. They first learn to handle basic properties of absolute value and roots. They further study the equations of an unknown first and second degree, while devoting many hours to the study of first and second degree inequalities. Finally, they study arithmetic and geometric progress, as well as basic functions. In the second grade of senior high school, students learn a new way of solving a linear system, while being able to solve non-linear systems. They study for the first time monotonicity and extremes of a function, as well as exponential function and logarithms. While there is a great development of trigonometry and further study of polynomials. In parallel with science-oriented mathematics, students are taught the basic theory of vectors. Finally, in the last year of senior high school, in recent years, the algebra section in the textbook that deals with tables is out of the curriculum and subject matter.

Despite, the significance of Algebra, difficulties begin quite earlier even before school entry [4]. Recent research findings in young children indicated that child-associated variables (such as gender and chronological age) are correlated both to early numeracy. Moreover, the number of children within the families 
emerged as a significant factor and indicated a positive correlation with respect to increasing the number of children in a family [5]. A more detailed investigation could shed light on the phenomenon.

\subsubsection{Geometry}

Geometry is one of the oldest branches of mathematics. Both the Babylonians and the Egyptians, as well as the ancient Greeks, dealt in depth with spatial relations, with axioms and definitions for straight lines, planes, and solids. When Plato spoke of mathematics, he essentially meant geometry, giving it almost metaphysical roots. The leading work on geometry to date is Euclid's work "The Elements", a total of thirteen books, which contain 121 definitions, 5 requests, 9 common concepts and 465 sentences. The first four books along with the sixth talk about flat geometry. A typical example is the Pythagorean theorem, which is contained in the first book. The fifth book together with the seventh to the tenth book has to do with number theory, as well as with numbers, mainly fractions, which represent lengths of straight sections. In it, concepts such as that of prime numbers, explicit and implicit are introduced. Finally, the third part, consisting of the eleventh book to the last, talks about stereometry, giving, among other things, ways to calculate their areas and volumes. As mentioned, in the Evidence and specifically in the first book Euclid presents five requests. These are the following:

$>$ From any point to any other we can only bring one straight line.

Any finite line can be extended continuously.

$>$ From any center and with any radius we can draw a circle.

All right angles are equal to each other.

$>$ If a straight line intersects two others and forms angles inside and on them, less than two right angles, then the two straight lines, if extended indefinitely, will meet the side where the angles that are less than two right angles are.

The fifth request is also called a parallel request because it is equivalent to the following sentence "From a point outside the line we can bring exactly one parallel to the line". It is also what seemed less convincing than the rest and this has caught the attention of mathematicians since the time of Euclid for two millennia. Two thousand years later the great mathematician Gauss (1777-1855) was the first to conclude that there could be a new geometry based on all the demands of Euclid and to replace the fifth request of the parallels "From a point outside a line we can bring more than one-line parallel to the line". He himself never published the above notes, but they were found after his death thus giving prestige to the announcements of the discovery made almost simultaneously, albeit independently, by the mathematicians Bolyai in 1832 and Labachevsky in 1829. This geometry is now perfectly acceptable and known as excessive geometry. Around the same time, also great mathematician Riemann (1826-1866) discovered another non-Euclidean geometry, the so-called Elliptical geometry. The most important of the theorems of Elliptical geometry was that "there are no parallel lines" [6]. 
In secondary education in our country, students come into contact exclusively with Euclidean geometry. More specifically in high school students initially learn basic knowledge, ie definitions of geometric concepts, such as: the different types of angles as well as the relationships between them, straight and straight sections, circle, lines and angles in relation to them, as well as study of symmetry. In the second grade of high school students are able to find the area of basic geometric shapes as well as the volume of basic solid shapes. They also learn to apply the Pythagorean theorem, the basic definition of trigonometric numbers, and study the circle. By finishing high school students are able to know more about trigonometry, as well as learn the criteria of triangle equality, Thales' theorem and its applications and finally find similar polygons and triangles.

In senior high school, students study Euclidean geometry a little more. In the first grade, they study the triangles again and more completely. Their species, their equality criteria, triangular inequality, etc. Then they study the parallel lines, studying among others the five requests of Euclid. Finally, they study the registered geometric shapes, the resulting relationships and the conditions for their registrability. In the second grade, students are confronted with the study of geometry for the last time in their school life, since geometry is not taught in the last grade of senior high school. In Euclidean geometry of general education, students are asked to study basic proportions either through straight sections or in the light of Thales' and bisector theorems, but also similarities. As well as metric relations, areas of flat shapes and measurement of a circle with both known types of calculation of elements of the circle, as well as with an inscribed regular polygon. Finally, they study the properties of the line in the plane. In addition to these, students who have followed a positive studies orientation study in more depth the straight line and conical sections, i.e. the circle, the lack, the exaggeration and the parable.

\subsubsection{Analysis}

Another key area of mathematics is analysis. It is a more modern branch since it was founded in the late 17th century independently of Leibniz and Newton. Basic fields of analysis are infinite calculus, ie the sum of differential and integral calculus, real, arithmetic and complex analysis, as well as the functions of differential equations and measure theory. Finally, part of the analysis is topology and metrics, along with basic concepts such as boundaries and convergence of functions, sequences and of course real numbers. Analysis has contributed greatly to the development of technology, assisting in areas such as quantum mechanics, hydrodynamics, thermodynamics and engineering. As well as in meteorology and biology.

In high school students have little contact with the field of mathematical analysis. This is because the more abstract nature of analysis requires greater age development on the part of children, but also an introduction to symbolic mathematics which is achieved mainly through algebra. Teaching about analysis in high school, apart from of course the basic concepts of real numbers, is limited 
to the elementary study of basic functions that fall into the field of elementary algebra.

In senior high school students are gradually introduced to mathematical analysis. In the first grade, students are taught arithmetic and geometric progress, as well as studying graphs and basic functions. In the second grade, general education students are introduced to the concepts of monotony, extremes and symmetry of a function. They also study exponential function and logarithms. Students who have chosen the orientation of the positive sciences, on the other hand, as we saw in the chapter on geometry, study geometrically, but also the functions of basic conic sections. Finally, in the last grade of senior high school, students are introduced to basic concepts of analysis. Students in Mathematics and Statistics will be taught the basic rules of derivation, while their classmates who choose the positive orientation will first study the limits and continuity of a function, then enter the differential calculus by studying the derivative, the derivative, average value, local extremes, curvature and inflection points of a function, as well as the asymptotic ones along with the De l' Hospital rule. Finally, students are taught integral calculus, they learn to calculate indefinitely and some integrals, as well as to calculate the area of a flat passage with the help of integral.

\subsubsection{Statistics}

Statistics is the mathematical tool for extracting results from our existing data. Fisher (1890-1962) is considered the father of modern statistics and gave the most common and well-known definition of what statistics is. Statistics, then, is a set of principles and methodologies for:

$>$ the design of the data collection process, which is called experimental design.

$>$ their concise and effective presentation and is called descriptive statistics.

$>$ the analysis and drawing of corresponding conclusions, methods that make up inferential statistics or inferential statistics.

Statistics are now widely used in almost all areas of human activity. Scientific fields such as physics, health and technology, as well as the humanities, law and social sciences use and have fully integrated statistics into their processes. Statistics in the economic field, of course, play a major and decisive role.

In high school students in the second grade come into contact with basic concepts of statistics such as sample, population, mean and median. They also learn basic graphical representations of presentation of results. In the third year of high school, students learn the basics of designing Venn diagrams, as well as the basics of sets. Then they are taught the sample space and what is the probability, as well as the meaning and the way of calculating the probability.

In senior high school with the exception of a report made in the first grade, on their sets and operations, students meet again the statistical science in the third grade in the course Mathematics and Statistics. There they learn basic concepts and methods of data collection. Methods for presenting statistical data are then taught, such as statistical tables, frequency distribution tables and cumulative 
frequencies, as well as frequency distribution graphs, observation grouping, frequency histogram and finally frequency curves. They also study the location and dispersion measures. Students then enter the world of probability by first studying the sample space and contingencies as well as the operations with contingencies. Finally, the definition and properties of relative frequency are taught, as well as the classical and axiomatic definition of probability.

\section{Learning Disabilities}

In recent decades, the expansion of compulsory education, the development of the knowledge-based character of the school, as well as the development in the field of child psychology and developmental psychology, as well as special education have led to the observation of cases of children without obvious mental retardation that couldn't meet standard school requirements. The finding that there are people who have difficulty learning in the predetermined and established way has been the birthplace of the scientific field of learning disabilities. Learning disabilities (LDs), as a scientific field, are a newly established field. This sector is characterized by stages of search, turmoil and redefinition in its short course so far trying to define, establish and integrate in the context of wider special education and training. The approaches that prevailed from time to time, formed corresponding definitions and generally influenced the evolution and development of the field. In the early phase of the field, the approach was medical-centric, but as the field evolved, the approach became more psychoeducational, which resulted in the further development of the field. The attempt to establish a definition of learning disabilities followed the changes in the field from time to time.

However, the definition used to date is the one formulated in 1990 by the $\mathrm{Na}$ tional Joint Committee on Learning Disabilities and is as follows: "Learning disabilities are a general term that refers to a heterogeneous group of disorders that manifest with significant learning difficulties and use of intuition, speech, reading, writing, reasoning or mathematical skills. These disorders are inherent and are thought to be due to dysfunction of the central nervous system. They can occur throughout life. They may also coexist with problems in self-regulatory behavior, social perception and social interaction, but by themselves these characteristics do not constitute learning difficulties. Although they may occur in combination with other types of deficiencies (such as sensory deficits, mental retardation, severe emotional disturbances) or with external influences such as cultural differences, inadequate or inappropriate teaching, they are not the result of these factors or effects" [7] [8].

A not insignificant percentage of students face difficulties related to school learning, which lead to poor school performance. These difficulties are perceived by the teacher, even by classmates, during the educational process. Apart from significantly affecting the learning ability and performance of each student, they also affect his behavior inside and outside the classroom, but at the same time 
they do not manifest in the same way in all children.

According to the World Health Organization, a school failure can be due to one of the following categories of causes or a combination of them [9]:

1) Mental problems, such as neurotic states, child psychosis, behavioral disorders, adaptation reactions, etc.

2) Special delays in development, such as dyslexia, dysnumeracy, speech development disorder, etc.

3) Problems from the mental level, such as mental retardation, borderline intelligence states, and even high intelligence.

4) Medical conditions, such as vision and hearing disorders, motor difficulties, chronic diseases, epilepsy, etc.

5) Psychosocial situations, such as cultural deprivation, bilingualism, inadequate living conditions, unfavorable family conditions, etc.

As mentioned above, learning disabilities are not expressed in the same way by all children who face them. Primarily because there is a wide range of diverse forms of learning disabilities. The most common learning difficulty is dyslexia. Students with dyslexia can experience many forms of difficulty, such as in word decoding, spelling, fluency and rhythm of their oral speech, as well as comprehension and written expression. Students with difficulty decoding words have difficulty reading, which in turn leads to poor reading performance. However, children with developmental speech disorders, such as dysphasia, children with lower mental capacity, or children with emotional or social problems may also have difficulty reading.

Another learning problem that one may face is that of dysgraphia. These people usually have a combination of difficulties in their writing skills, as evidenced by grammatical errors, punctuation errors, poor text organization, multiple spelling errors, too poor calligraphy and inconsistent keeping spaces between words. As well as these people have difficulty thinking and writing at the same time. But a spelling or grammar disorder should not automatically be included in a dysgraphia diagnosis without considering other factors. This is getting more important since recent data shows that teachers in the COVID-19 situation believe that they do not receive the adequate support to overcome difficulties [10].

Dyscalculia is another learning difficulty, which includes difficulties such as learning mathematical concepts, retaining mathematical data, as well as the difficulty of solving a mathematical problem due to difficulty in recognizing logical sequences of information received and problems in understanding the chronological sequence of events. People with dyscalculia may also have difficulty understanding mathematical symbols, telling the time, or understanding basic mathematical concepts, such as negative numbers.

Another learning disability is the special speech disorder or dysphasia. These individuals show a significant retardation of speech development for no apparent reason and have difficulty understanding and using both written and spoken 
language. Developmental coordination disorder or dyspraxia is used interchangeably with the term clumsy child syndrome. To date, there is no commonly accepted definition and clearly defined pattern of behavior, but opinions converge on the fact that it is a disorder with the main feature of severe impairment in the development of motor coordination that is not explained by other known underlying causes. However, to talk about clumsy child syndrome, any motor problems that may have developed in children must have a significant impact on daily activities, as well as school performance. Lansdown [11] proposed the description of deficient classes as follows:

- rough mobility difficulties, such as walking, running, climbing, etc.

- problems with fine mobility, such as finger coordination difficulties, etc.

- visual-motor problems, such as difficulties in catching the ball, in writing, in painting etc.

He described the common characteristics of children with clumsy child syndrome, stating that the skills exist, but lag behind in quality. Also children usually understand the mistakes in the programming, but they cannot correct them. He went on to say that these children have difficulty in pacing and assessing their strength required to successfully complete a process. While finally he mentioned that they generally have a slower rhythm than the other children and have difficulty in understanding organizational concepts, such as the shapes before-after, up-down etc.

Another disorder that can be detected is that of Attention Deficit Hyperactivity Disorder. Its main characteristics are intense and persistent hyperactivity, intense impulsivity, short-term attention, emotional arousal, low resistance to failure, and sometimes antisocial behavior. To talk about a disorder, the symptoms must be present for at least six months, usually appearing in children of preschool age and above, while receding into adolescence but leaving the learning and social problems [12].

Finally, in our country there are several dozen medical-pedagogical services that have the ability to evaluate and certify people with learning disabilities. These include departments of general hospitals, mental health structures and Centers for Differential Diagnosis, Diagnosis and Support of Special Educational Needs.

\subsection{Instructional Intervention}

As mentioned earlier, students with learning disabilities may find it difficult to learn in the traditional way. This is why the most important skill they need to acquire is to learn how to learn. Teachers if they have knowledge of different methods and strategies can apply them and thus facilitate the learning of these children, which can help not only to improve school performance, but also to make life easier overall.

A strategic intervention in education, therefore, is a specific process aimed at helping children with learning disabilities to improve in the area of their needs, 
which can vary (Lee) and affect the student coding process [7]. This can change the student's emotional state, for example it can reduce stress or enhance the process of capturing and encapsulating new information by the student.

Historically, we see different approaches to defining the relevant concepts. In 1979 Alley and Deshler gave to learning strategies the definition of technical principles or rules that facilitate the acquisition, handling, integration, storage and retrieval of information in different situations and contexts. They are adequate, effective and organized steps or procedures used in learning, recall and performance situations. That is, it is a long-term process that helps children with learning disabilities to be able to first understand and then learn and apply new data, in addition to being able to enrich their existing knowledge with new ones in a conceptual way. That is, to be able to add to an idea that already knows new features, to be able to separate the data from each other as well as to operate in an abstract way when required, so that there is a generalization of knowledge and it is recalled in different situations and frameworks that need to be implemented.

Naus and Ornstein, in 1983, as Siegler reports, defined strategies as "cognitive or behavioral activities that are subject to voluntary subject control and are used to enhance memory performance." Children use strategies in all phases of memorization, that is, as they encode material, as they store it, and finally as they retrieve it. Many age-related memory improvements reflect the acquisition of new strategies, the cultivation of existing strategies, and the application of existing strategies to new situations [13]. Moreover, difficulties in early numeracy acquisition frequently are attributed to memory deficiencies [3].

Deshler and Lenz, a decade later than Alley and Deshler, said that approaching an individual at a job is called strategy. The approach includes both cognitive and behavioral elements, which guide the student's performance and the evaluation of his work. In addition, the strategy should help the student to evaluate the results of the effort that has been applied during the execution of the strategy [14]. Also according to Swanson strategy is defined as a set of responses organized to solve a problem.

It would be appropriate here to clarify that today when we talk about strategic interventions in education, the aim is solely to strengthen the academic process, in order to improve the student's academic performance and not his behavior. In more detail, they aim at a specific challenge and are specific and standardized, as they last a certain and specific period of time and are evaluated in intermediate stages. Despite the formalistic nature of the learning interventions, they are at the same time flexible since there is a possibility to change them when it is found that the intervention strategy followed is not effective.

So, to summarize, we are talking about learning strategies which are a set of steps aimed at helping children with learning disabilities to improve what is difficult for them, have a specific goal and are designed so that the teacher and the school in general can control progress achieved. So the teachers of both general 
and special education enter the classroom with two goals, the first is what the student should learn and the second is how he will learn it.

\subsection{Response to Intervention (RTI)}

The growing knowledge we had about learning disabilities, as well as their pedagogical approach, led to the need for a new, alternative model for the identification, identification and ultimately support of students with LD. This task was completed by Fuchs in 1995 by defining the framework of this model, which he called "Response to Instruction and Intervention" (RTI). The model is nothing more than the process of continuous assessment and determination of how a child with learning disabilities responds to specially designed teaching and intervention.

RTI is a method of early detection and diagnosis, while preventing and supporting students with learning disabilities, with the aim of dealing with school failure. The application of RTI had as an important result the release of the diagnosis of MD from the traditional psychometric diagnostic methods. A key point in RTI's implementation in 2004 was the US decision to enact a law proposing the universal application of RTI by all schools, in order to identify students with $\mathrm{MD}$, as well as to support students with school difficulties regardless of which special category they belong to [12].

RTI seems to be a model aimed at realizing that the education system can teach all children effectively. A crucial parameter is the timely intervention, in order to avoid the possibility that the difficulties will be consolidated and can no longer be overcome. On this it is necessary to apply a multi-level model, which will be analyzed below. A problem management model can be used as a basis for decision making at each level. In addition, interventions can be applied immediately, which are scientifically substantiated based on scientific research findings. Finally, monitoring the students' progress will play a key role, as the progress data will give feedback to the teaching and will guide the teachers to make decisions [12].

\subsection{Types of Intervention Strategies}

Teaching students is a challenge that teachers are called upon to face on a daily basis. Especially when we talk about students with learning difficulties, the standard teaching is very likely to be insufficient. There are different types and types of strategic intervention in teaching used in general schools, RTI schools, and special schools. This chapter will present and analyze these formulas, with a look at their application to the teaching of mathematics.

\subsubsection{Explicit Instruction}

Explicit teaching is a method of teaching skills or concepts to students, using direct and structured teaching. It helps in the crystallization of the content of the respective lesson, models the process of implementation of a task and gives time for practice. The teaching here should be absolutely clear, the steps should be 
clear and complete, it should not leave gaps or raise questions as to which direction should be followed in solving a problem. Explicit teaching is a well-researched, effective teaching method that can be applied to all levels of education and to all categories of students.

Explicit teaching consists of four steps, since the goal is clear first. Initially a model is created with clear explanations, presented in the same way that students will use it, with steps that are clearly written, concise and consistent, focusing on the most critical points of the process. The thought process that is done by the students when they are called to solve the problem is then given verbally. Then comes the time to practice what has been taught to see if there has been an understanding of the solution steps, as well as to find any "gaps" in the model presented. Supervised practice is especially important to ensure the success of the whole process. Once the understanding in the steps is confirmed, the practice should now become independent, without the teacher's supervision. It would also be important to review the process again after the students' independent practice. Finally, there is a constructive critique and comments during the control of the students' practice so that the mistakes are corrected immediately and are not established as the right process. (Greene)

\subsubsection{Corrective Feedback}

Corrective feedback is a common method used in teaching. It can be either formal or informal and is offered to the student by the teacher so that the former has knowledge of his performance. It aims to encourage students to continue or to try to correct their mistakes. The feedback can be immediate, ie at the time of solving an exercise, it can be by correcting a written exam by filling in comments and information above in addition to the grade or it can be for a longer period of time, such as the grades each quarter students receive.

Feedback should be objective, reliable and specific. It should also be detailed, ie it should be clear what the student did correctly and what did not. There the student will need to understand what needs to be improved and how to do this. In addition, it must be done frequently, not occasionally in order to give a complete and objective picture of the student's progress, while finally it must concern exclusively the student's academic progress.

\subsubsection{Concrete Representational Abstract-CRA}

The CRA teaching model is a three-step approach and consists of direct explicit teaching, with problem modeling, guided and independent practice of students and immediate feedback to them. It also includes representation strategies so that students can move from physical objects to conceptual knowledge [15]. The approach followed is gradual and sequential, each level is based on the concepts taught in the previous level. It promotes overall conceptual understanding, procedural accuracy and agility, using multi-sensory learning techniques to introduce new concepts and ideas [16]. Let's see in more detail and separately what each stage of the CRA includes: 
Stage 1: During this stage of teaching, three-dimensional physical objects are used so that students can use touch to help as they learn a new concept [17]. Using touch increases a student's likelihood of remembering the process it took to solve a problem [18].

$>$ 2nd stage: In this stage students learn to draw and manipulate two-dimensional drawings to represent the same concept that through the touch of three-dimensional objects they had defined in the previous stage. The manipulations performed in the first two stages help students to rationalize concepts and processes in logical steps and comprehensible definitions [16]. In case of a high degree of students' difficulty it is possible to create iconographic representations to help solve the problem [18].

> Stage 3: In the last stage, students are taught how to translate two-dimensional drawings into conventional mathematical symbols and through them be able to solve problems [17].

\subsubsection{Concrete Manipulatives}

This method uses physical objects, which students can touch and move. Thus students have the opportunity to explore them and to be able to elicit their qualities and characteristics. Through these the teacher can promote new concepts and ideas or strengthen and establish the already known concepts, but which have not been encapsulated. As students' progress, teachers expect their handling to go beyond just handling tools. The purpose is to allow children to understand the ideas and concepts, otherwise they will remain abstract and they will not be able to manipulate them. As the student grows, the knowledge he has acquired is more complete and will not be based only on the sense of touch, but this memory can be recalled so that manipulations can now be done through imaginary objects and not physical ones [19].

\subsubsection{Visual Representation}

Another strategy that research has shown to help students learn abstract mathematical concepts and solve problems is illustrative representations. More than a simple image, a pictorial representation is an accurate representation of mathematics that involves creating and shaping models that reflect mathematical information [20].

An important feature is that the visual representations are flexible. They can be used at different levels and types of mathematical problems, as well as take different forms depending on the problem we are called to deal with and what they should represent. They can be diagrams, which can be iconographic or schematic and help to organize and clarify the steps to solve a problem. Or it could be a ruler, a straight line showing the order and relationship between the numbers.

\subsubsection{Multisensory Approach}

The multi-sensory approach is a teaching method that involves linking more 
than one sense at a time. It includes the use of visual, auditory, kinesthetic and tactile stimuli and more. May include the senses of taste and smell. The multi-sensory approach can enhance the student's memory and learning ability, because it can stimulate their brain more intensely and better to delve deeper into the object of study. Although each lesson does not necessarily use all five senses of a child at the same time, most lessons that use this approach use educational materials so that students do not approach it with just one sense [21].

All students, whether they have learning difficulties or not, benefit from using the multi-sensory approach in a lesson because if a student has the opportunity to learn something using more than one sense, the new data is more likely to be remembered and encapsulated. Multi-sensory teaching helps children to use the ways in which they feel most comfortable learning, making the necessary conceptual connections and forming memories. As well as having more flexibility in delivering the knowledge they have received. In conclusion, the multi-sensory approach respects and recognizes that different children learn differently, thus meeting their learning needs, giving them the opportunity to succeed [21].

\section{Aim of the Research}

Education and consequently the process of teaching and learning is a fundamental good of our society that must be provided to all. For this reason, a benevolent society should aim to prevent educational and social exclusion of people who find it difficult to cope with the curriculum pursued in education in a formal way. This may include people with disabilities or people with learning disabilities.

In the present work we focus on the second category of people and look for the best or the best intervention methods for the various fields of mathematics. And specifically we focus on students who are in secondary education, ie high school and senior high school students and we look for solutions for the whole range of mathematics that students are taught.

Although there is a large literature on methods of strategic intervention in students with learning disabilities in primary education, this is not the case for secondary education. As desirable as it is to have any learning disabilities diagnosed early and students receive the support they need to improve their performance, this does not mean that they are eliminated over time. Thus, it is necessary to know their proper management during the period of High School and Lyceum.

The present study therefore expects to present a dual result, both theoretical and applied. Theoretical because its future reader can be informed about the theoretical framework around learning difficulties and strategic interventions. But also because he will be aware of the most common problems and areas in the teaching of mathematics that cause confusion and difficulty in being understood by learners. Finally, it is of practical interest because it will present the optimal strategic intervention for each sub-sector of mathematics separately, as this emerges through the review of research. 


\section{Research Questions}

Research questions played a major role in the creation, design and implementation of this work.

What are the strategic interventions implemented in the process of teaching mathematics to students with learning disabilities?

$>$ Are there more effective intervention strategies compared to others? And if so what are they?

$>$ Does the specific intervention strategy prevail in all areas of mathematics where students have difficulty or is there an optimal strategy for each area?

> In what end do students face greater difficulty in coping with learning difficulties? And to what extent were they helped after the intervention?

Are there areas of mathematics or mathematical subjects that, while taught in secondary education, we do not identify research that contains them as a subject of study?

These questions guided all stages of the Systematic Bibliographic Review, as mentioned earlier.

\section{Research Results}

The present work aims to conduct a Systematic Bibliographic Review-SBR in order to find the effectiveness of strategic interventions for teaching mathematics to students with learning difficulties in secondary education. The previous chapter delineated the theoretical framework through which our research was implemented. This chapter will describe the findings of this search.

\section{Selection of Studies}

The systematic bibliographic search identified and recorded two hundred and forty-four surveys (244) from the search in databases, of which two hundred and twenty-nine (229) were unique records, while fifteen (15) were duplicates. At the same time, forty-three (43) studies were identified through other sources (related research work of a researcher, recording of research from other reviews, etc.). Of these, twenty-nine (29) were single recordings, while fourteen (14) were duplicate recordings. A total of two hundred and fifty-eight (258) unique publications were recorded.

Then the screening check of the surveys was done. Initially the title screening was done. During this process, fifty-three (53) inquiries, all from database searches, were rejected because they did not meet the exclusion criteria. Reasons such as that they were not studies with some strategic intervention included, were not the subject of mathematics or were students below the age limit we are studying. The abstracts were then checked (abstract screening). A total of one hundred fifty-four (154) inquiries were rejected during this process, for reasons similar to those previously reported. Of these, one hundred and forty-two (142) came from primary research, while twelve (12) from secondary research through other sources. A total of two hundred and seven (207) surveys were rejected and 
fifty-one (51) surveys remained for further study.

The control process continued with the eligibility test by studying the full texts of the surveys (full-text screening). During this process, a total of thirty (30) studies were rejected for reasons related to the above. Of these, eighteen (18) come from primary research and twelve (12) from secondary research. A total of twenty-one (21) surveys met the inclusion criteria. Of these, six (6), four (4) from the primary investigation and two (2) from the secondary investigation, were omitted in the composition of the results due to incomplete recording of data and data.

Finally, twelve (12) and three (3) studies from the primary and secondary research respectively, i.e. a total of fifteen (15) were included in the composition of the results.

\section{Discussion}

The school is the same as the basic pillars of effort from the personal timers for the applications that require the thumbnail of the photo for the students, the questions and the questions we want to ask, to make them appear in a different video as to the user name, the type of family. In this way we achieve the primary user, who is the one required to obtain the dissemination of information from, different use is given to the children that I should not do with the required specific destinations.

Focusing on our main goal, we find that in recent years more and more in the educational system of our country is embracing the various learning peculiarities. Aiming to strengthen this effort taking place in our schools, we have worked to find key answers to questions that plague us.

It was clear from the research included in the synthesis of results that the effectiveness of a strategic educational intervention tends to change depending on the mathematical subject we are called to teach. Nevertheless, there was absolute stability in the effectiveness of specific strategies, and a variety of combinations were presented. The study of these results is a major part of the present work, together with the recording of the mathematical fields, in which it was found that students most often face difficulties in their learning. Also pointed out are the shortcomings that we have identified in the research search for mathematical objects.

For the needs of the research, the protocol of the systematic bibliographic review in electronic databases was followed with a methodological structure followed according to PRISMA CKECKLIST [22].

Studying the research that was included, we were led to draw conclusions about our initial research questions. Then a separate and detailed answer to the individual questions will be given, combining the inclusion research.

\section{Conclusions per Research Question}

1) What are the strategic interventions implemented in the process of teaching 


\section{mathematics to students with learning disabilities?}

In our research we included fifteen surveys. All involved controlled strategic intervention during the teaching of various mathematical subjects to students with learning difficulties. In twelve of them, explicit instruction and its various models are used. Specifically, we encounter a method that is based on the interests of students (interest-based method) and is part of an approach to explicit teaching, cognitive strategy. Cognitive strategy is found in two other studies, in which we identify the meta-cognitive strategy, an approach that aims at the self-knowledge of the knowledge that is necessary for the effective solution of problems, while at the same time guiding and regulating the cognitive processes in solving a problem [23]. We also encounter two more models of explicit teaching, the graphic organizers and the schema based. In fact, the last of the models we mentioned was identified in three surveys. One study also used the explicit inquiry routine, an approach that combines explicit teaching with CRA. Finally, regarding explicit teaching, we find the use of culturally responsive Instruction and modified culturally responsive Instruction.

Another strategic approach we encountered many times was that of concrete-representational-abstract (CRA). Specifically, we identified it in five surveys, either as such or partially and often in combination with other methods, such as explicit teaching, concrete manipulative tools, visual representations and specifically representational drawings and the graphic organizer. On the other hand, the method of hand tools was identified, apart from its combination with CRA. In one study there was an autonomous method of intervention, while in another it was combined with virtual manipulatives, thus constituting a multisensory approach. Finally, the corrective feedback that is part of the explicit teaching, and not only, was found to play an important independent role in two studies.

2) Are there more effective intervention strategies compared to others? And if so what are they?

In the present research there is no analysis and synthesis of results through statistical means and tools. As a result, we cannot determine with absolute accuracy the degree of effectiveness of strategic interventions in relation to a predetermined mathematical field. Nevertheless, we obtain clear and documented approaches from the systematic literature review in response to the present research question.

From the research we came up with for their inclusion in the composition of the results, only three emerged, which clearly had a comparison of the effectiveness of two strategic interventions in relation to a specific mathematical object. In these three, the results showed a superiority of the CRA method over the explicit teaching mainly, but also over the RA. At the same time, it becomes quite clear that targeted strategic interventions are clearly more effective than general traditional teaching, as there has been an increase in student performance, a fact that became known through the comparison of pre-diagnostic and post-diagnostic 
tests.

Analyzing the results of the research, it was found that a group of students with learning difficulties managed to reach the same level of performance as students without learning difficulties, after teaching them verbal and mathematical problems, based on their personal interests. A result that is deeply essential if we consider that our goal is to gradually lead to the inclusion of students with learning difficulties in the general formal class as having the same opportunity to learn. Another very useful conclusion we draw from the research study is that students tended to prefer to use the methods taught during the intervention as the mathematical problem they were asked to solve became more difficult and complex.

It is also pointed out that in the vast majority of cases there is a better long-term performance of students, as well as the possibility of generalizing the method taught during teaching with intervention, in other cases of mathematical problems. Contradictory results were presented in relation to the long-term performance of students after the teaching intervention with the method of cognitive approach. Explaining this it is useful to point out that from the first to the second research there was a period of nineteen years. During this time the research team developed and improved the method of intervention, based on the same principles, making it more complete. This resulted in the method presented and applied in the subsequent research yielding clearly better results and achieving them to have long-term validity. A similar situation with a modification of the intervention method in the implementation of the research occurred during the use of culturally responsive teaching. The change in method brought immediate positive results to the students.

3) Does the specific intervention strategy prevail in all areas of mathematics where students have difficulty or is there an optimal strategy for each area?

Through the review we found that there was a different educational approach of students with learning disabilities depending on the mathematical subject on which the research focused each time to study. Here, too, the clear superiority of explicit teaching is evident. As it is found capable of bringing about positive performance results of students with learning difficulties in various fields of mathematics. But it has always been combined with other strategic interventions as well as optimized models.

Starting with algebra, a field with a higher frequency of occurrence as the object of research, we identify seven researches which had as a field the mathematical verbal problems. There were four approaches to solving them. Through three studies, the impressively high efficiency of their solution-based method was found, an enriched approach to explicit teaching. This method seemed to help students in the long run, as well as to enable students to generalize this method to other mathematical objects than what was taught during the intervention. In addition, it was found that students seemed to seek to use this method more as the difficulty of the exercise they were asked to solve increased. This 
leads us to the conclusion that students were first able to understand and clarify the method, on the other hand it proves the effectiveness of the method in facilitating students with learning difficulties to solve verbal math problems.

The interest-based method was then identified in research, ie the provision of additional cognitive teaching strategy, an approach of explicit teaching to students with learning difficulties that will help them process the content of verbal mathematical problems. Through this method, students with learning difficulties were able to eliminate the differences in performance they had with their classmates without learning difficulties. In two more studies, which studied mathematical speech problems, the cognitive and post-cognitive approaches were used.

As we mentioned shortly before, in the concluding answer to the second research question, in one study the results were not promising either in terms of students' understanding of the method or in terms of its retention at a later level. While in the research that followed, the research team, maintaining the principles of the cognitive and post-cognitive approach, developed a model for solving verbal mathematical problems, which it named "Solve It". That is, a complete strategic routine consisting of seven cognitive processes (reading, paraphrasing, visualization, hypothesis, assessment, calculation and control) and corresponding self-regulation strategies (self-teaching, self-questioning and self-monitoring). This time the results of the research were positive and supportive, with the additional feature that the positive effect had a long-term effect. Thus it can be recorded as an effective method for students with learning difficulties in solving mathematical verbal problems. Finally, for mathematical speech problems, the EIR solution method was identified, a combined approach of explicit teaching with CRA. The method proved to be effective in solving the problems of a variable, but showing a small performance gain at a later time, but with the ability of students to apply it in a variety of mathematical areas.

4) In the end, what make it more difficult for students to cope with learning disabilities? And to what extent were they helped after the intervention?

The purpose of this research is the systematic bibliographic review of research that has implemented teaching through strategic intervention in a mathematical subject for students with learning difficulties. Each researcher or research team chose the mathematical object, which was the field of study of their research, having as a fixed selection criterion what is difficult for these students. The vast majority of the research we include for drawing conclusions has the field of algebra, with verbal and mathematical problems monopolizing the interest, since almost half of the research is studied.

It has been reported that students with learning disabilities fail to achieve an adequate conceptual understanding of the basic concepts that govern the functions and algorithms used to solve problems involving whole and logical numbers [24]. Problem solving should be defined as the ability to create a wide variety of possible strategies, the ability to evaluate the possible consequences of 
each strategy and the ability to design a logical sequence for the implementation of useful strategies [25]. The most widely used problem-solving strategy is the general four-step solution process (reading, designing, solving, and controlling). Unfortunately, this process may not make it easy to solve problems for students with learning disabilities, especially when the conceptual and procedural knowledge of specific areas is underdeveloped [26]. On this occasion, research was conducted in order to be able to help students with learning difficulties in solving verbal mathematical problems. There was indeed an impressive increase in yield, with the possibility of maintaining this profit as well as its generalization.

Students also found it difficult to implement the long division solution algorithm. In this case, too, the reinforcement of teaching with hands-on tools helped the students to understand the solution algorithm and the steps required. Another mathematical subject that seems to be very difficult for students, and especially those with learning difficulties, is what surrounds the equivalent fractions. The reason for this difficulty may be the difficulty of these students to understand concepts in general [27].

To solve this, the goal is to try to be able to understand the rules governing equivalent fractions, as well as to achieve a connection with real life, in order to gain interest through their applicability, but also to gain an understanding of their meaning. Students appeared to be significantly enhanced after CRA intervention in combination with the use of hand tools, while they had lower performance gains in area fractions. The students then found it difficult to solve equations. The reason for this difficulty may be the unsuccessful transition of students from arithmetic to symbolic algebra. As well as the flexibility of handling the dual interpretation of symbols, sometimes as concepts and sometimes as processes. Although algebra evolves from arithmetic, there is a learning gap. In other words, knowledge of arithmetic is necessary but not sufficient for the success of algebra [28]. Objects such as linear algebraic equations, systems of linear equations, multiplication equations for area calculation and algebraic transformation equations are difficult for students to deal with.

As for the systems of equations, the students after the intervention showed a better understanding of the concepts, while they seemed to be able to follow the process of solving them with greater ease. At the same time, their preference for the method of organizing graphs, which was taught during the intervention, became apparent, as the level of difficulty of solving the system increased. In the other three math subjects, students increased their performance after the intervention using CRA. Finally, it was found difficult to solve problems related to area and perimeter calculation. The students were strengthened in their performance both in the short and long term in the specific subjects of geometry, but mainly in the problems of area calculation, after the intervention with the use of hands-on and virtual training tools.

5) Are there areas of mathematics or mathematical subjects that, while taught in secondary education, we do not find research that contains them as a subject 
of study?

In the inclusion criteria we defined and applied in this paper there was no restriction in a mathematical field or subject. They aimed at the whole range of mathematics that students are taught in the curriculum in secondary education. The results of the systematic literature review, however, did not contain entire areas of mathematics, while even in the areas identified there were deficiencies in mathematical objects. In addition, another very important observation concerns the age range of the students who participated in the surveys included for the synthesis of results. Students over the age of fifteen were observed in only two studies. So based on the Greek data we would say that almost all the surveys concern high school students, while we have little data to include for high school students. Naturally, this greatly affected the cognitive objects we identified and recorded.

Starting from the areas we identified during the review and specifically from algebra, no research was identified on explicit numbers and negative numbers. In fact, the transition of students from natural numbers to other sets of numbers and especially to negatives is a great challenge for students, since they are faced with counting numbers, which cannot be done by counting a set of objects. Also no research was found with the subject of study the inequalities, the methods of factorization, as well as trigonometry. Finally, as far as algebra is concerned, there has been no research involved in absolute value, roots and polynomials.

Continuing with the field of geometry, it was found that there are only a few researches with a relevant subject, which concern the problems of finding area and perimeter. Thus, research on the basic knowledge and definitions of geometry and the calculation of the volume of solid shapes is not included. In addition, no research has been identified that includes as a subject the study of triangles and what it includes, objects such as the criteria of triangle equality, the Pythagorean theorem and much more. There were also no data on conical sections and proportions. Finally, it should be mentioned that there was a research in the synthesis of results which included the whole mathematical spectrum that is taught to students at the age of fourteen on average. This range included objects in algebra and geometry. However, due to the peculiarity of this research-it concerns cultural diversity of students-and because we do not have the results separately for each mathematical subject, we cannot draw a conclusion that concerns all students with learning difficulties.

As a result of the age restriction observed in surveys that met the criteria for inclusion, areas such as analysis and statistics are not represented at all. Thus important mathematical objects such as functions, limits, continuity, derivation and integrals are not part of any research from the review. Simultaneous statistical objects such as Venn diagrams, probabilities and concepts such as dispersion, and frequency are also not found in the research we are studying.

\section{Conclusions}

Mathematics and the education system in general must be accessible to all. 
Bearing in mind the importance of acquiring mathematical knowledge and taking into account the difficulties faced by many students with learning difficulties in mathematics, there is an urgent need to incorporate educational strategies that are effective in order to help high school students with learning difficulties have access to the general education mathematics program in a meaningful way.

The educational interventions used by teachers to improve the academic performance of students with learning disabilities are influenced by various factors. Factors influencing educational change include the government's policy decisions on education, the prompting of experts on learning disabilities and, of course, the results of intervention surveys. The results of this review provide a number of educational recommendations, including:

1) Include a graded teaching sequence in teaching to help students move to abstract levels of mathematical comprehension by following this concrete tangible teaching sequence [29] [30].

2) Teachers teach to achieve comprehension with a layout-based teaching strategy to help students represent the underlying mathematical structures and lead to solution [31].

3) Teaching using the CRA method in combination with organization of graphs is effective when included in the teaching of algebra to high school and high school students as a method of differentiated teaching [32].

4) Classrooms should be designed in such a way as to increase students' interactions with new concepts that reinforce their prior knowledge [18].

5) Teachers should continue to explore practical and iconographic approaches that effectively represent the mathematical processes that lead to productive and efficient mathematical skills associated with conceptual comprehension [18].

\section{Suggestions and Restrictions}

The field of learning disabilities is a complex and multifaceted issue. Although many researchers around the world have studied this subject, there are still items that can be suggested as future research.

From the very first stages of the present research it was found that the vast majority of research that studies learning disabilities has as participants, students who are of primary age in primary education. Subsequently, a relatively small number of studies concern high school students. In addition, few surveys were found to involve higher education students. So it is a first proposal as a future research, the implementation of educational interventions for students older than the age of twelve.

Probably as a result of the above observation and as described in the answer to the fifth research question, it is found that no research in the mathematical fields of analysis and statistics was identified, while we did not distinguish research in the field of algebra and geometry. Therefore, a further study is proposed in order to intervene effectively in the teaching of these areas.

Also, from the review emerged researches which almost all of them were im- 
plemented in the USA. No relevant intervention research was identified in a Greek school. There would be a great breath of development in the field of learning difficulties in our country, if a scientifically substantiated research intervention in mathematics was implemented in students with learning difficulties of secondary education in a Greek school. Finally, in the present work, other research questions could be added, such as how much the number of participants influenced the intervention and how the intervention sections were structured. While the framework for the inclusion of research could be tightened and a meta-analysis of the results could be carried out, for a more valid conclusion.

However, as humanity evolves more and more questions will arise around the improvement of the quality of life and the provision of goods and services, so more and more research will be carried out in search of answers to these questions.

\section{Acknowledgements}

This study would not have been possible without the contribution of numerous people. We would like to thank the educational staff who took part in the research.

\section{Conflicts of Interest}

The author declares no conflicts of interest regarding the publication of this paper.

\section{References}

[1] Charitaki, G., Tzivinikou, S., Stefanou, G. and Soulis, S.G. (2021) A Meta-Analytic Synthesis of Early Numeracy Interventions for Low-Performing Young Children. SN Social Sciences, 1, Article No. 105. https://doi.org/10.1007/s43545-021-00094-w

[2] Shapiro, S. (2000) Thinking about Mathematics. The Philosophy of Mathematics. Oxford University Press, Oxford.

[3] Charitaki, G., Baralis, G., Polychronopoulou, S., Lappas, D. and Soulis, G.S. (2015) Difficulty in Learning to Count or Effect of Short-Term Memory Deficiency in Mathematical Abilities? International Journal of Innovation and Research in Educational Sciences, 2, 60-62.

http://www.ijires.org/index.php/issues?view=publication\&task=show\&id=56

[4] Charitaki, G., Baralis, G., Polychronopoulou, S., Lappas, D. and Soulis, G.S. (2014) Early Numeracy in Children with Down's Syndrome in Greece. Psychology, 5, 1426-1432. https://doi.org/10.4236/psych.2014.512153

[5] Charitaki, G., Baralis, G., Polychronopoulou, S., Lappas, D. and Soulis, S.G. (2014) Factors Related to Numerical Ability of Children with Down's Syndrome. The International Journal of Early Childhood Learning, 21, 1-17.

http://ijlecl.cgpublisher.com/product/pub.256/prod.48 https://doi.org/10.18848/2327-7939/CGP/v21i01/48429

[6] Boyer, C.B. and Merzbach, U.C. (1997) The History of Mathematics. 2nd Edition, G. A. Pneumatikos Publications, Athens.

[7] Tapouridou, D. (2014) Learning Strategies for Children with Learning Disabilities. 
Unpublished Master's Thesis, Aristotle University of Thessaloniki, Thessaloniki.

[8] Tzouriadou, M. (2011) Learning Difficulties. Issues of Interpretation and Dealing. Promitheus Publications, Thessaloniki.

[9] Markovitis, M. and Tzouriadou, M. (1991) Learning Difficulties. Theory and Action. Promitheus Publications, Thessaloniki.

[10] Tzivinikou, S., Charitaki, G. and Kagkara, D. (2020) Distance Education Attitudes (DEAS) during Covid-19 Crisis: Factor Structure, Reliability and Construct Validity of the Brief DEA Scale in Greek-Speaking SEND Teachers. Technology, Knowledge and Learning, 1-19. https://doi.org/10.1007/s10758-020-09483-1

[11] Woodard, M. and Lansdown, R. (1988) Language and Communication. In: Richman, N. and Lansdown, R., Eds., Problems of Preschool Children, Wiley, Chichester, 1-18.

[12] Tzivinikou, S. (2015) Learning Difficulties. Didactic Interventions. Association of Greek Academic Libraries National Technical University of Athens, Athens.

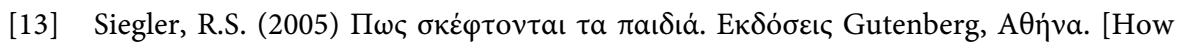
Children Are Thinking. Athens: Guttenberg.]

[14] Lenz, B.K. (1992) In the Spirit of Strategies Instruction: Cognitive and Metacognitive Aspects of the Strategies Intervention Model. In: Vogel, S.A., Ed., Educational Alternatives for Students with Learning Disabilities, Springer-Verlag, New York, 141-158. https://doi.org/10.1007/978-1-4613-9168-5 6

[15] Sealander, K., Johnson, G.R., Lockwood, A.B. and Medina, C.M. (2012) Concrete-Semiconcrete-Abstract (CSA) Instruction: A Decision Rule for Improving Instructional Efficacy. Assessment for Effective Intervention, 30, 53-65. https://doi.org/10.1177/1534508412453164

[16] Witzel, B.S., Riccomini, P.J. and Schneider, E. (2008) Implementing CRA with Secondary Students with Learning Disabilities in Mathematics. Intervention in School and Clinic, 43, 270-276. https://doi.org/10.1177/1053451208314734

[17] Miller, S.P. and Kaffar, B.J. (2011) Developing Addition with Regrouping Competence among Second Grade Students with Mathematics Difficulties. Investigations in Mathematics Learning, 4, 24-49. https://doi.org/10.1080/24727466.2011.11790308

[18] Witzel, B.S. (2005) Using CRA to Teach Algebra to Students with Math Difficulties in Inclusive Settings. Learning Disabilities. A Contemporary Journal, 3, 49-60.

[19] Douglas, H. (2000) Inductive Risk and Values in Science. Philosophy of Science, 67, 559-579.

[20] Van Garderen, D. and Montague, M. (2003) Visual-Spatial Representation, Mathematical Problem Solving, and Students of Varying Abilities. Learning Disabilities Research \& Practice, 18, 246-254. https://doi.org/10.1111/1540-5826.00079

[21] Morin, A. (n.d.) Multisensory Instruction: What You Need to Know. https://www.understood.org/en/school-learning/partnering-with-childs-school/inst ructional-strategies/multisensory-instruction-what-you-need-to-know

[22] Moher, D., Liberati, A., Tetzlaff, J. and Altman, D. (2009) Preferred Reporting Items for Systematic Reviews and Meta-Analyses: The PRISMA Statement. PLOS Medicine, 6, e1000097. https://doi.org/10.1371/journal.pmed.1000097

[23] Montague, M. (1992) The Effects of Cognitive and Metacognitive Strategy Instruction on the Mathematical Problem Solving of Middle School Students with Learning Disabilities. Journal of Learning Disabilities, 25, 230-248. https://doi.org/10.1177/002221949202500404 
[24] Baroody, A. and Hume, J. (1991) Meaningful Mathematics Instruction: The Case of Fractions. Remedial and Special Education, 12, 54-67. https://doi.org/10.1177/074193259101200307

[25] Edeh, O. and Hickson, L. (2002) Cross-Cultural Comparison of Interpersonal Problem Solving in Students with Mental Retardation. American Journal on Mental Retardation, 107, 6-15. https://doi.org/10.1352/0895-8017(2002)107<0006:CCCOIP >2.0.CO;2

[26] Hutchinson, N.L. (1993) Effects of Cognitive Strategy Instruction on Algebra Problem Solving of Adolescents with Learning Disabilities. Learning Disability Quarterly, 16, 34-63. https://doi.org/10.2307/1511158

[27] Hiebert, J. (1985) Children's Knowledge of Common and Decimal Fractions. Education and Urban Society, 17, 427-437. https://doi.org/10.1177/0013124585017004006

[28] Witzel, B., Smith, S. and Brownell, B. (2001) How Can I Help Students with Learning Disabilities in Algebra? Intervention in School and Clinic, 37, 101-104. https://doi.org/10.1177/105345120103700205

[29] Butler, F.M., Miller, S.P., Crehan, K., Babbitt, B. and Pierce, T. (2003) Fraction Instruction for Students with Mathematics Disabilities: Comparing Two Teaching Sequences. Learning Disabilities Research \& Practice, 18, 99-111. https://doi.org/10.1111/1540-5826.00066

[30] Witzel, B.S., Mercer, C.D. and Miller, M.D. (2003) Teaching Algebra to Students with Learning Difficulties: An Investigation of an Explicit Instruction Model. Learning Disabilities Research \& Practice, 18, 121-131. https://doi.org/10.1111/1540-5826.00068

[31] Xin, Y.P., Jitendra, A.K. and Deatline-Buchman, A. (2005) Effects of Mathematical Word Problem-Solving Instruction on Middle School Students with Learning Problems. The Journal of Special Education, 39, 181-192. https://doi.org/10.1177/00224669050390030501

[32] Strickland, T.K. and Maccini, P. (2012) The Effects of the Concrete-Representational-Abstract Integration Strategy on the Ability of Students with Learning Disabilities to Multiply Linear Expressions within Area Problems. Remedial and Special Education, 34, 142-153. https://doi.org/10.1177/0741932512441712 